\title{
BMJ Open Recreational drug use in the Oslo nightlife setting: study protocol for a cross-sectional time series using biological markers, self-reported and qualitative data
}

\author{
Trond Nordfjærn, Marit Edland-Gryt, Anne Line Bretteville-Jensen, Kristin Buvik, \\ Johanna Gripenberg
}

To cite: Nordfjærn T, EdlandGryt M, Bretteville-Jensen AL, et al. Recreational drug use in the Oslo nightlife setting: study protocol for a crosssectional time series using biological markers, selfreported and qualitative data BMJ Open 2016;6:e009306. doi:10.1136/bmjopen-2015009306

- Prepublication history for this paper is available online. To view these files please visit the journal online (http://dx.doi.org/10.1136/ bmjopen-2015-009306).

Received 6 July 2015 Revised 19 November 2015 Accepted 27 January 2016

CrossMark

The Department of Alcohol, Drug and Tobacco Research, Norwegian Institute of Public Health, Oslo, Norway

\section{Correspondence to} Dr Anne Line BrettevilleJensen;

anne.line.bretteville-jensen@ fhi.no

\section{ABSTRACT}

Introduction: Recreational drug use in the nightlife setting carries the risk of many negative consequences, such as violence, injuries, aberrant driving and sexual risk-taking. The aim of this study is to investigate recreational drug use and user characteristics among people visiting licensed premises, for example, nightclubs and bars, by using self-reports and biological markers. Staff of licensed premises will be asked to report drug use observations. Further, by using qualitative data, we will examine the motives, consequences and culture associated with recreational drug use. An additional aim is to compare self-reported drug use with oral fluid test (OFT) results in order to validate the different measurement methods in this context.

Methods and analyses: Data collection will be conducted among patrons $(n=1000)$ outside licensed premises. On consent, patrons will be asked to anonymously complete a questionnaire, a breath alcohol concentration test and an OFT. Patrons who report use of recreational drugs in the previous 12 months will be asked to leave their contact information for a subsequent qualitative in-depth interview $(n=30-40)$. Staff from licensed premises $(n=500)$ will be invited during Responsible Beverage Service Training to participate in an anonymous survey. Survey data will be analysed by univariate and multivariate statistical methods and the oral fluids will be analysed for a large number of drugs using biochemical methods. Cohen's $\kappa$ will be used as a measure of agreement between self-reported drug use and OFT. In-depth interviews will be coded in HyperRESEARCH and analysed using an inductive approach. Data collection will be repeated on a biannual basis until at least 2020 , allowing for examination of trends in recreational drug use.

Ethics and dissemination: This study has been approved by the Regional Committee for Medical and Health Research Ethics. Results will be disseminated in research journals, conferences and the media.

\section{Strengths and limitations of this study}

- A rich supply of data from a high-risk group in a high-risk setting, including biological measures, self-reports and in-depth qualitative data.

- Both cross-sectional and time series data.

- Likely to yield results with high relevance for methodological development in the field.

- Provides a better understanding of the culture associated with recreational drug use.

- Causal interpretations not possible.

\section{INTRODUCTION}

Recreational drug use in the nightlife setting is potentially a public health problem that requires targeted interventions. Short-term consequences include increased risk of violence, injuries, aberrant driving and risky sexual behaviour. ${ }^{1}{ }^{2}$ Adverse long-term effects may be the development of somatic or mental disease, ${ }^{3}$ occupational activity dropout (eg, work or education) and ultimately addiction. Alcohol reduces prefrontal cortical activity, which may in turn increase the propensity to experiment with recreational drugs. Licensed premises (eg, nightclubs, bars) are common environments for young adults to be introduced to recreational drugs and to initiate use under the influence of alcohol. ${ }^{3}$ The concurrent use of two or more substances, that is, polysubstance use, increases the risk of negative public health effects even further. ${ }^{45}$

Traditionally, recreational drug use among young adults (ie, individuals aged 1830 years) has been examined by surveys conducted in schools/universities or in the general population. ${ }^{6} \quad{ }^{7}$ These approaches may not have effectively reached the target 
population because young adults with high recreational drug use are not necessarily present in school/university settings or may fail to respond to population surveys. Young adults with a strong propensity to use drugs are likely to actively seek out the nightlife setting. ${ }^{3}$ Therefore, it could be more feasible to reach out to these individuals in this context. Furthermore, previous surveys have tended to only include self-reported data, which are potentially skewed towards socially desirable responses. Also, the fact that response rates to surveys have been decreasing could challenge the ecological validity of results.

Recent advances in the field of drug testing allow for an unobtrusive and efficient collection of oral fluid tests (OFT) to examine the presence of a range of drugs. This method has significant advantages over selfreporting, where both over-reporting and underreporting are common. OFT can detect drugs such as cocaine, amphetamines, cannabis and the new psychoactive substances (NPS). ${ }^{8}$ NPS are psychoactive drugs that could represent a public health risk similar to the more traditional drugs. Some NPS are not (yet) prohibited by law in several countries. ${ }^{9} 10$

Our study will have a specific focus on the use of NPS and a broad initial test repertory of 122 different drugs, including 'classic' drugs (eg, cannabis, cocaine and amphetamines).

There are relatively few studies which have combined OFT and self-reports of recreational drug use. Some of these studies suggested considerable under-reporting on questionnaires when compared to OFT results. ${ }^{11}$ Previous work regarding recreational drug use in nightlife settings has also tended to focus on special events such as electronic music dance events ${ }^{11-13}$ or has been carried out in specific settings, such as gay clubs. ${ }^{14}$ Further, few previous studies have incorporated multiple sources of data including qualitative in-depth interviews, and the majority of previous studies were conducted at one point in time, which excludes the possibility for examinations of temporal trends in recreational drug use. There are also few studies including licensed premises staff. The present study will advance previous studies by investigating recreational drug use in a large pool of different licensed premises, including people participating in the nightlife setting and staff enrolled in Responsible Beverage Service (RBS) Training. Thus, we will approach the phenomenon by using multiple sources of data, that is, self-reporting, biological and qualitative data, coupled with multiple measurement waves (time series).

\section{Research objectives}

The main aim of our study is to examine recreational drug use and the characteristics of users in the Oslo nightlife setting. We also aim to investigate motives, consequences and the culture surrounding recreational drug use in this setting. An additional aim is to compare self-reported drug use with OFT results in order to test the validity of different methods for measuring recreational drug use in this context. The findings are expected to yield information that will be used to establish adequate community-based interventions in the nightlife setting.

\section{METHODS AND ANALYSIS}

During the study, we will obtain four types of data: (1) cross-sectional survey data from patrons outside licensed premises, (2) cross-sectional biological data from patrons outside licensed premises, (3) cross-sectional survey data from staff at licensed premises, and (4) data from individual qualitative in-depth interviews (see also figure 1). Data collection 1-3 will be repeated biannually until 2020 .

\section{Procedures}

\section{Cross-sectional patron survey}

Cross-sectional data will be collected from a sample of patrons close to the entrance of strategically selected licensed premises. A modified portal survey methodology will be used, where data will be collected once for each participant, instead of twice (on entering and exiting licensed premises) as was done in previous work. ${ }^{15} 16$ Our aim is to increase our knowledge about recreational drug use in the nightlife setting, rather than knowledge about drug intake inside licensed premises, which is why we have chosen this modified method.

To establish a sample that covers the broad spectrum of the nightlife setting in downtown Oslo, we will obtain a complete list of licensed premises from the municipality administration. The list will be reviewed by an expert group of relevant authorities. Premises that clearly do not match the inclusion criteria will be excluded. A number of licensed premises will be selected by the experts using the following criteria: (1) popular and frequently visited licensed premises with peak hours between 23:00 and 3:00 am, (2) premises with a geographic spread in central downtown Oslo, (3) a focus on the 'party factor', for example, dancing and high alcohol consumption, and (4) premises with different profiles and patron characteristics. A sort of 'ethnographic mapping, ${ }^{17} 18$ will also be used to ensure that the clubs match the criteria. Similar approaches have been used to select licensed premises for studies in Europe, ${ }^{17} 19$ the $\mathrm{USA}^{20}$ and Australia. ${ }^{21}$ For the biannual follow-up studies, the same inclusion criteria will be used to select licensed premises.

A total of 20 research assistants will conduct the data collection. They will receive training in recruitment and on the study procedures. The assistants will be divided into four groups (five people in each group) and the data collection will be conducted from 23:00 to 4:00 on Fridays and Saturdays.

Data collection stations are going to be placed close to the entrance of the selected licensed premises and an imaginary line will mark out a selection zone. As patrons 


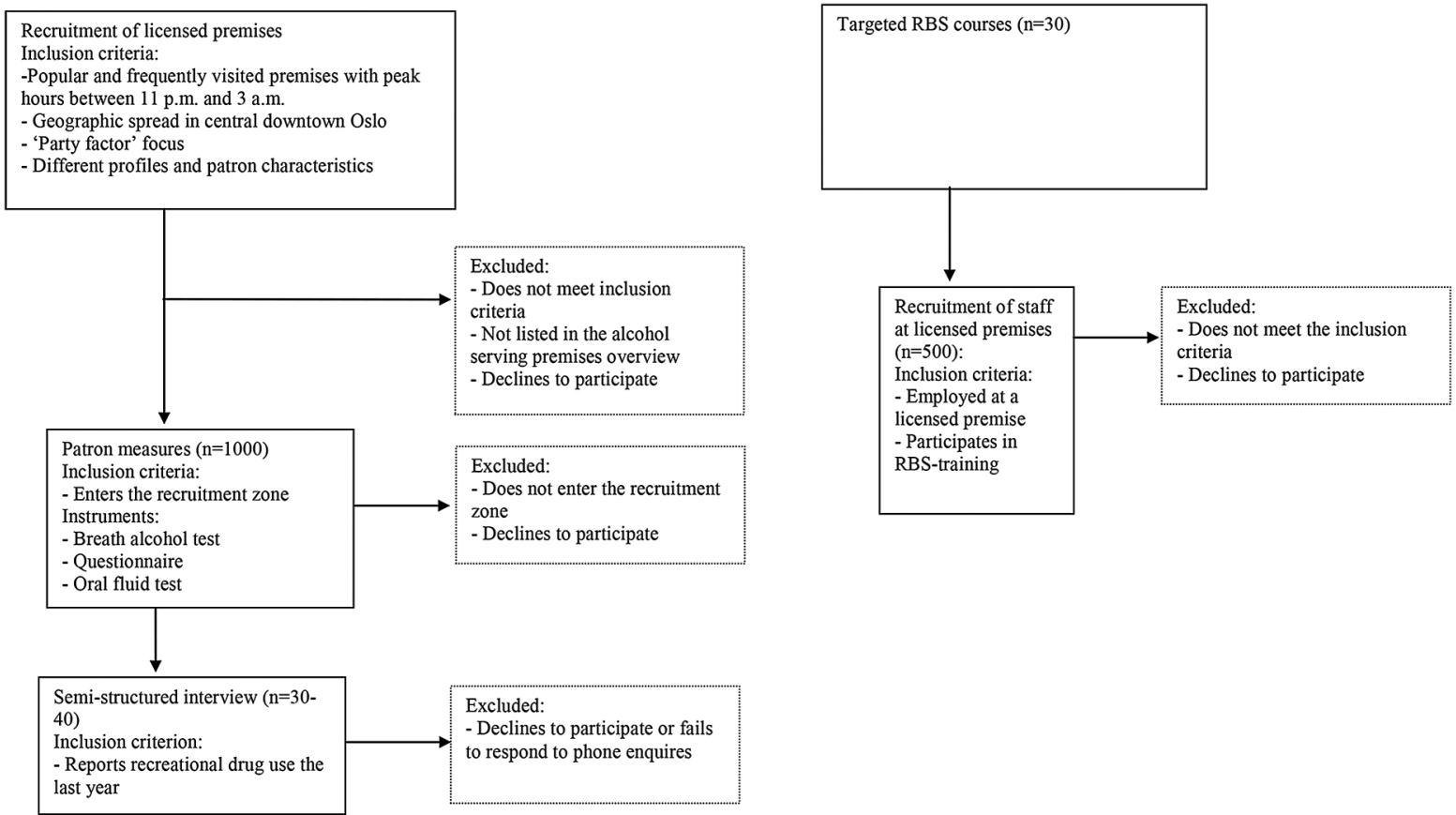

Figure 1 Flow chart of the study design. RBS, Responsible Beverage Service.

cross the line, a research assistant will approach them and invite them to participate. They will offer patrons an informed consent statement, verbally and in writing. When the person is part of a group, the whole group will be asked to participate. Previous research has found the refusal rate to be high when single people from a group are invited to participate. ${ }^{19}$

Once patrons consent to participate in the anonymous study, they will be asked to give a breath alcohol test (BAC) and then be asked to complete a questionnaire and provide an OFT. Research assistants will help participants to fill out the questionnaire, if requested. We will record the gender and estimated age of persons who decline to participate. The questionnaire will contain a unique serial number for each respondent and the OFT samples will be given a corresponding number. The BAC level measured by a breathalyser will be noted on each individual questionnaire. Since the results from the breathalyser will be instantly available, the participants will be informed about their BAC levels should they request it. Throughout the data collection process, the participants will be able to withdraw from the study. The questionnaires as well as the BAC tests, OFT procedures $(n=80$, respectively) and in-depth interviews $(n=5)$ will be pilot tested among relevant user groups before formal data collection starts.

\section{Cross-sectional staff survey}

Staff $(n=500)$ at licensed premises attending RBS training ( $n=30$ courses) will be asked to participate in an anonymous survey in a classroom setting. Norwegian municipalities arrange RBS training on a regular basis, where the majority of the participants are staff at licensed premises. Participants will complete the survey during RBS training. The survey will include sections about personal characteristics, participants' observations of drug use at their workplace, participants' drug use and their opinions on drug policy.

\section{Time series}

To establish time series data, we will repeat the survey biannually until 2020 using the same methods described above. That is, we will use the same criteria for selecting licensed premises and for recruiting participants. Further, we plan to keep the structure of the questionnaire unchanged, although we may have to slightly adjust the questions if drug use changes.

\section{Qualitative in-depth interviews}

The study will also include individual in-depth interviews $(n=30-40)$. We will ask study participants who report use of recreational drugs other than alcohol within the previous 12 months to voluntarily leave their phone number to be contacted later for an individual in-depth interview during the day. A similar method to recruit participants has been used elsewhere previously. ${ }^{17} 1822$ The patrons will be able to choose where and when they wish to be interviewed, though they can decline to participate at any time. They will receive a letter of confirmation, which will contain the name and contact details of the researcher. The patrons will be informed about the voluntary nature of the interviews, and that they can withdraw their consent and have their data deleted at any point in time. The interviews will particularly focus on the use of MDMA/ecstasy, cocaine, amphetamines and NPS, and informants who report use of these drugs 
will be purposefully selected for the interviews. We will obtain written consent for the interviews to be recorded. The interviews will be recorded by a digital recorder, and are expected to last for $1-2 \mathrm{~h}$. The participants will receive a compensation of about $32 € / 300$ NOK. The recorded interviews will be anonymously transcribed into text.

\section{Assessment}

Questionnaire

A questionnaire will be based on variables found to be important in previous work. ${ }^{11} 12$ The patron questionnaire will contain demographic items such as gender, age, region of birth, education and employment status. It will also include questions about the frequency of recreational drug use during the respondents' lifetime, the past year, the past month and the past $48 \mathrm{~h}$. The list of drugs will include alcohol, tobacco, cannabis, NPS (eg, synthetic cannabis, legal highs, spice, research chemicals, etc), amphetamines, cocaine, LSD, heroin, ecstasy/ MDMA as well as anabolic steroids. We will also include a dummy drug (MOP) in order to examine the validity of self-reporting and extent of over-reporting. The respondents will also be questioned about the age of onset for each drug. Further, the questionnaire will include variables such as the past year frequency of alcohol intoxication, the age of first alcohol intoxication experience and frequency of visits to licensed premises. In addition, the questionnaire will include a section to record the participants' BAC level yielded by the breathalyser.

Oral fluid will be collected from patrons using the Orasure Intercept Oral Fluid Drug Test. The device is composed of a sampling pad made of cotton on a plastic stick. The cotton pad is placed under the tongue for a few minutes to collect saliva. The cotton pad is then transferred to a plastic tube, which contains a buffer with a preservative. The samples will be kept cold $\left(2-8^{\circ} \mathrm{C}\right)$ until delivered to the analytical laboratory where they will be frozen. The sample will be analysed for a large number of drugs using liquid chromatography with tandem mass spectrometry detection.

For the pilot study, we will use an extended test repertory including 122 drugs covering the 'classic' drugs as well as the NPS (see table 1). The list of 122 drugs was based on the types of drugs found in Norway by the customs services as well as updated drug-monitoring information. The final test repertory will be derived from the drugs detected in the pilot study and the drugs from recent seizures by the Norwegian customs services. Before new data collection waves are conducted, the oral fluid screening repertory will be critically reviewed and possibly revised. The repertory will be updated with new drugs based on seizures by police and customs as well as updated expert information about new drugs detected in Norway. Patrons' BAC levels will be measured by using the Lion Alcolmeter 500, which is a high validity breathalyser.
The survey methodology that we will use to collect data from staff at licensed premises has been developed and tested by researchers in Sweden. ${ }^{19}$ Similar procedures and a slightly modified questionnaire will be used in this study. The survey will include four sections: demographics, respondents' own alcohol and drug use experience, respondents' attitudes towards recreational drug use and observed drug use among guests at licensed premises. Both patron and staff questionnaires will be translated to English and used for respondents without proficiency in Norwegian.

\section{Qualitative in-depth interviews}

A semistructured interview guide will be used to explore central themes regarding attitudes, motivations and experiences related to use of alcohol and recreational drugs, with particular focus on MDMA, ecstasy, cocaine, amphetamine and NPS. The patrons will be encouraged to speak freely, and the semistructured interview guide will ensure that two core topics are covered. The first topic will be motivation for recreational drug use. This section will delve into positive and negative psychological (eg, reducing distress and tension, enhanced positive mood, unpleasant psychological feelings of 'coming down' from the drug), social (eg, peer pressure and social enhancement), somatic (eg, increased physical performance) and contextual (eg, lack of alternative rewarding activities to drug use) factors underlying use. Open-ended questions regarding specific situations and states that might increase a patron's' tendency to use recreational drugs will also be included. This component will focus particularly on shared norms, values and beliefs between the individual and their peers (ie, cultural factors), which could be relevant to recreational drug use. The second core topic will focus on the positive and negative consequences of recreational drug use. This section includes questions related to perceived consequences of drug use on mental and somatic health, social relationships, and work and educational activities.

\section{Analyses}

Univariate analyses will be used to describe recreational drug use and the characteristics of users. Multivariate quantitative approaches, including regression analyses, will be undertaken to assess associations between a wide range of characteristics and drug use. Cohen's $\kappa$ coefficient will be applied to test the correspondence between self-reported use and results from OFT. We will use time series analysis to investigate trends in drug use over time. Independent samples $t$ tests and $\chi^{2}$ analyses will be conducted as appropriate to compare gender and age characteristics among respondents and non-respondents.

The qualitative data will be analysed using an inductive approach. ${ }^{23} 24$ This means that predefined themes from the interview guide will be set aside to search for emerging themes from the interviews that the researcher had not specifically looked for. The patterns, categories and themes will be built from the 'bottom-up', and 
Table 1 Analysis repertory for the study

\begin{tabular}{|c|c|}
\hline Drug class & Type \\
\hline Amphetamines & $\begin{array}{l}\text { Amphetamine } \\
\text { Methamphetamine } \\
\text { MDMA } \\
\text { PMA } \\
\text { PMMA } \\
\text { 2-Fluoroamphetamine (2-FA) } \\
\text { 3-Fluoroamphetamine (3-FA) } \\
\text { 4-Fluoroamphetamine (4-FA, PFA) } \\
\text { 4-Methylamphetamine } \\
\text { 4-Methylthioamphetamine } \\
\text { 4-Fluoromethamphetamine } \\
\text { 4-Methylmethamphetamine (4-MMA) }\end{array}$ \\
\hline Opiates/opioids & $\begin{array}{l}\text { Morphine } \\
\text { Codeine } \\
\text { Ethylmorphine } \\
\text { Oxycodone } \\
\text { Tramadol } \\
\text { Tapentadol } \\
\text { AH-7921 } \\
\text { Carfentanyl } \\
\text { Fentanyl } \\
\text { Methadone } \\
\text { Buprenorphine } \\
\text { 6-MAM }\end{array}$ \\
\hline Benzodiazepines & $\begin{array}{l}\text { Alprazolam } \\
\text { Bentazepam } \\
\text { Diazepam } \\
\text { Diclazepam } \\
\text { Etizolam } \\
\text { Flubromazepam } \\
\text { Fluntirazepam } \\
\text { Oxazepam } \\
\text { Clonazepam } \\
\text { N-desmethyldiazepam } \\
\text { Nitrazepam } \\
\text { Pyrazolam }\end{array}$ \\
\hline $\begin{array}{l}\text { Cannabis and } \\
\text { synthetic } \\
\text { cannabinoids }\end{array}$ & $\begin{array}{l}\text { THC } \\
\text { 5F-Apinaca } \\
\text { 5F-PB-22 } \\
\text { AM-2201 } \\
\text { AM-2233 } \\
\text { AM-694 } \\
\text { HU-210 } \\
\text { JWH-015 } \\
\text { JWH-018 } \\
\text { JWH-019 } \\
\text { JWH-073 } \\
\text { JWH-081 } \\
\text { JWH-122 } \\
\text { JWH-200 } \\
\text { JWH-203 } \\
\text { JWH-210 } \\
\text { JWH-250 } \\
\text { JWH-251 } \\
\text { MAM } 2201 \\
\text { PB-22 }\end{array}$ \\
\hline
\end{tabular}

Continued
Table 1 Continued

\begin{tabular}{ll}
\hline Drug class & Type \\
\hline & RCS-4 \\
RCS-4-C4 & RCS-8 \\
STS-135 & UR-144 \\
UR-144 Deg. \\
URB 754 \\
WIN 55.212-2 \\
XLR-11 Deg. \\
XRL-11
\end{tabular}

Cathinones Ethylcathinone

2-metylmetkatinon (2-MMC)

3-methylmethcathinone (3-MMC)

4-methylmethcathinone (4-MEC)

Methylone (bk-MDMA,

3,4-methylenedioxy- $\mathrm{N}$-methylcathinone)

Alfa-PVP

bk-MDDMA (dimethylone)

MDPV (methylenedioxypyrovalerone)

Pentedron

Phenethylamines $2 \mathrm{C}-\mathrm{C}$

$2 \mathrm{C}-\mathrm{I}$

2C-T-2

2C-E

2C-T-7

2C-P

2C-B

DOB

DOI

Bromo-DragonFLY

25C-NBOMe

25I-NBOMe

Butylone (bk-MBDB)

BDB (1,3-benzodioxolylbutanamine)

$\mathrm{N}, \mathrm{N}$-dimethyl-MDA

2,5-DMA (dimethoxyamphetamine)

Aminoindanes

2-Al (2-aminoindan) MDAI

(5,6-Methylenedioxy-2-aminoindane)

Tryptamines Dimethyltryptamine (DMT)

5-MeO-DMT

alfa-methyltryptamine

Piperidines/ 2-DPMP (Desoxypipradrol)

pyrrolidines

Ethylphenidate

3,4-CTMP

(2,4-Dichloromethylphenidate)

Piperazine

pFPP (p-fluorophenylpiperazine 1-(4-

derivatives

fluorophenylpiperazine)

1-benzylpiperazine (BZP)

TFMPP

mCPP (1-(3-chlorophenyl) piperazine)

Arylalkylamines

$6-A P B$

5-APB

$5-I T$

6-APDB

Miscellaneous Methoxetamine

Xylazine 


\begin{tabular}{ll} 
Table 1 Continued & \\
\hline Drug class & Type \\
\hline & Desomorphine \\
& Phenmetrazine \\
& Metaoxedrine (phenylephrine) \\
& Harmin \\
& Salvinorin A \\
& Tapentadol \\
& Homoamphetamine \\
& (3-amino-1-phenylbutane) \\
& Methiopropamine (MPA) \\
& Ketamine \\
& LSD \\
& Zolpidem \\
& Zopiclone \\
& Cocaine
\end{tabular}

APB, Aminopropylbenzofuran; Deg, Diethylene glycol; FLY, 3C-Bromo-Dragonfly; IT, 5-(2-Aminopropyl)indole; MAM, Monoacetylmorphine; MDDMA, Hydrochloride; MDMA,

Methylenedioxymethamphetamine; PFA, para-fluoroamphetamine; PMA, para-Methoxyamphetamine; PMMA, para-Methoxy-Nmethylamphetamine; RCS, Research chemicals; STS, (N(adamantan-1-yl)-1-(5-fluoropentyl)-1H-indole-3-carboxamide; TFMPP, Trifluoromethylphenylpiperazine;THC,

Tetrahydrocannabinol; UR, TMCP-018, KM-X1, MN-001, YX-17; URB, (6-methyl-2-[(4-methylphenyl)amino]-1-benzoxazin-4-one); WIN, (R)-(+)-[2,3-Dihydro-5-methyl-3-(4-morpholinylmethyl)pyrrolo [1,2,3-de]-1,4-benzoxazin-6-yl]-1-napthalenylmethanone; XLR, 5 "-fluoro-UR-144.

organised into increasingly more abstract units of information. ${ }^{23}$ The qualitative in-depth interviews will be analysed after the transcribed text is coded in the HyperRESEARCH software. Some interviews will be coded by two different researchers to ensure that the themes are coded in the same way and to strengthen the validity in the study.

\section{Ethics and dissemination}

The study has been approved by the Regional Committee for Medical and Health Research Ethics (application No. 2014/192). The survey and biological markers will be collected anonymously, and written informed consent will be obtained for the semistructured interviews only. The qualitative in-depth interviews will be conducted during the day, and so the participants will most likely not be under the influence of any substance when giving their written consent. Outside the licensed premises, an informed consent statement will be presented verbally and also offered in writing to all participants. Signatures will not be collected in order to maintain confidentiality. Identifying information will not be captured, and so it will not be possible for respondents to withdraw their consent after their data are collected and collated. During the process, however, the respondents will be free to retract their consent and have their response deleted.

For the qualitative in-depth interviews, ethical issues will be especially important to consider, as themes may be sensitive. It will be essential to acknowledge how the participants experience the interview situation, and at the end of the interview the researcher will always ask the participant how he or she felt about the interview experience. The participants will be given the option to see a clinical psychologist, free of charge if they wish to do so.

There are also situations in the data collection outside the licensed premises that potentially could raise ethical issues. Many of these issues have been raised and discussed in the application for the study to the Regional Committee for Medical and Health Research Ethics. One issue is the fact that many of the participants in this study will be under the influence of drugs and/or alcohol at the time we meet them outside the licensed premises. Therefore, it will be important to train the research assistants in how to approach potential respondents and how to deal with plausible situations that can occur. If the research assistants discover someone who is extremely intoxicated and at risk of harming himself/ herself or others, they will be trained to call emergency care, such as the police or ambulance services. Each station outside the licensed premises will have a team leader, and this person will have a specific responsibility for the security of the research team.

The results from the study will be disseminated in highly regarded international peer-reviewed journals, research conferences, reports and to the mass media.

\section{Strengths and limitations}

Owing to the potentially adverse short-term and longterm effects of drug use in the nightlife setting, it is crucial to obtain more knowledge about recreational drug use and the characteristics of users. Earlier studies in this context have mostly relied on the survey method. This study will investigate the use of recreational drugs in the Oslo nightlife setting using a combination of selfreporting, biological markers and qualitative data. To the best of our knowledge, this is the first study that examines recreational drug use in the nightlife setting using this combination of different data collection methods, and recruiting participants from a large pool of licensed premises with different characteristics. The results may help derive community-based interventions to reduce use and harm.

The study design has several strengths including triangulation between data sources and a large sample size reflecting the nightlife setting in central downtown Oslo. Our study will also help to determine the feasibility of using biological samples for future nightlife research. Use of biological samples may reveal more accurate results than self-reported data. The use of qualitative data will allow in-depth investigations of the cultural contexts where drugs are used and also provide important information about motivation and perceived consequences to be incorporated into potential interventions.

Possible limitations of this study include the crosssectional nature of data, which does not allow for conclusions about causality or temporal relations between variables. However, we will mainly use quite stable 
characteristics (eg, demographics) linked to the relevant outcomes. The fact that individuals might be intoxicated when they complete the questionnaire warrants cautious interpretation of the data. This limitation is mitigated by the inclusion of biological measures, which will yield objective data about recreational drug use.

Further, given the large number of NPS appearing on the illicit market and the rapid changes in availability, it is possible that the oral fluids testing will miss some NPS being used by the participants. As mentioned earlier, the drugs that will be tested for will be determined by the results from the very broad spectrum of drugs used in the pilot test samples and also by the list of drugs being confiscated by police and customs in recent years. The likelihood of overlooking many NPS should be limited, as we would expect that a frequently used drug would be confiscated by the authorities at some point. Also, we will ask the participants to name the new psychoactive drugs they have used. The same procedure will be used in the follow-ups to ensure that the oral fluid samples are examined for the most relevant drugs.

\section{Implications for interventions and future policy}

This study will be the first to provide data on recreational drug use in the Oslo nightlife setting. It will also identify characteristics among individuals who are more likely to use large amounts of a range of drugs, enabling targeted interventions. If recreational drug use turns out to be substantial, the findings may facilitate collaboration work between the health authorities and the nightlife industry to establish adequate countermeasures. A general tendency in the nightlife context in Oslo and elsewhere has been to implement measures aimed at reducing alcohol consumption, and there has been less focus on recreational drug use. For example, there have been studies involving professionally trained actors to act intoxicated, in order to study the frequency of alcohol service to seemingly intoxicated patrons. ${ }^{25-27}$ Similar research for which actors pretend to be intoxicated by recreational drugs has only been conducted in Sweden. ${ }^{28}{ }^{29}$ The results from our study will be able to provide the basis for planning and implementing drug prevention programmes aimed at patrons of licensed premises.

Contributors TN, ALB-J, ME-G and JG designed the study. KB contributed to the design of the study. TN wrote the study protocol with substantial critical input from all co-authors. All authors read and approved the final version of the manuscript.

Funding This work is carried out and mainly funded by The Department of Alcohol, Drug and Tobacco Research, Norwegian Institute of Public Health. The Norwegian institute of Public Health funds personell resources related to oral fluid analysis.

\section{Competing interests None declared.}

Ethics approval The study has been approved by the Regional Committee for Medical and Health Research Ethics (application 2014/192).

Provenance and peer review Not commissioned; externally peer reviewed.

Open Access This is an Open Access article distributed in accordance with the Creative Commons Attribution Non Commercial (CC BY-NC 4.0) license, which permits others to distribute, remix, adapt, build upon this work non- commercially, and license their derivative works on different terms, provided the original work is properly cited and the use is non-commercial. See: http:// creativecommons.org/licenses/by-nc/4.0/

\section{REFERENCES}

1. Bellis MA, Hughes $\mathrm{K}$, Bennett $\mathrm{A}$, et al. The role of an international nightlife resort in the proliferation of recreational drugs. Addiction 2003;98:1713-21

2. Grann M, Fazel S. Substance misuse and violent crime: Swedish population study. BMJ 2004;328:1233-4.

3. Gripenberg Abdon J. Drug use at licensed premises-Prevalence and prevention. Stockholm, Sweden: Karolinska Institutet, 2012. http://publications.ki.se/xmlui/handle/10616/40951

4. Lynskey MT, Agrawal A, Bucholz KK, et al. Subtypes of illicit drug users: a latent class analysis of data from an Australian twin sample. Twin Res Hum Genet 2006:9:523-30.

5. Medina KL, Shear PK. Anxiety, depression, and behavioral symptoms of executive dysfunction in ecstasy users: contributions of polydrug use. Drug Alcohol Depend 2007;87:303-11.

6. Bauman A, Phongsavan P. Epidemiology of substance use in adolescence: prevalence, trends and policy implications. Drug Alcohol Depend 1999;55:187-207.

7. Vedøy TF, Skretting A. Ungdom og rusmidler. Resultater fra spørreskjemaundersøkelser 1968-2008 [Adolescents and substances. Results from surveys]. Oslo, Norway: Norwegian Institute for Alcohol and Drug Research, 2009.

8. Øiestad EL, Johansen U, Christophersen AS. Drug screening of preserved oral fluid by liquid chromatography-tandem mass spectrometry. Clin Chem 2007;53:300-9.

9. New psychoactive substances review. Report of the expert panel. https://www.gov.uk/government/uploads/system/uploads/attachment data/file/368583/NPSexpertReviewPanelReport.pdf (accessed 18 Dec 2015)

10. United Nations Office on Drugs and Crime (UNODC). NPS New Psychoactive Substances. https://www.unodc.org/documents/drugs// printmaterials2013/NPS_leaflet/WDC13_NPS_leaflet_EN_LORES. pdf (accessed 18 Dec 2015).

11. Gripenberg-Abdon J, Elgan TH, Wallin E, et al. Measuring substance use in the club setting: a feasibility study using biochemical markers. Subst Abuse Treat Prev Policy 2012;7:1-10.

12. Miller BA, Furr-Holden CD, Voas RB, et al. Emerging adults' substance use and risky behaviors in club settings. J Drug Issues 2005;35:357-78.

13. Miller BA, Holder HD, Voas RB. Environmental strategies for prevention of drug use and risks in clubs. J Subst Use 2009;14:19-38.

14. Measham F, Wood DM, Dargan PI, et al. The rise in legal highs: prevalence and patterns in the of use of illegal drugs and first- and second-generation 'legal highs' in South London gay dance clubs. J Subst Use 2011;16:263-72.

15. Voas RB, Furr-Holden D, Lauer $E$, et al. Portal surveys of time-out drinking locations: a tool for studying binge drinking and AOD use. Eval Rev 2006;30:44-65.

16. Miller BA, Furr-Holden D, Johnson MB, et al. Biological markers of drug use in the club setting. J Stud Alcohol Drugs 2009;70:261-8.

17. Watters JK, Biernacki P. Targeted sampling: options for the Study of Hidden Populations. Soc Probl 1989;36:416-30.

18. Ravn S. Managing drug use in Danish club settings: a normalized enterprise? Young 2012;20:257-76.

19. Gripenberg-Abdon J, Wallin E, Andréasson S. "The club against drugs" program in Stockholm, Sweden: two cross-sectional surveys examining drug use among staff at licensed premises. Subst Abuse Treat Prev Policy 2011;6:1-8.

20. Miller BA, Byrnes HF, Branner AC, et al. Assessment of club patrons' alcohol and drug use: the use of biological markers. Am J Prev Med 2013;45:637-43.

21. Duff C. Party drugs and party people: examining the 'normalization' of recreational drug use in Melbourne, Australia. Int J Drug Policy 2005:16:161-70.

22. Järvinen M, Demant J, Østergaard J. Stoffer og Natteliv [Drugs and nightlife] (in Danish). Copenhagen: Hans Reitzels Forlag, 2010.

23. Creswell JW. Qualitative inquiry \& research. Choosing among five approaches. 2nd edn. Thounsand Oaks: Sage Publications, Inc, 2007.

24. Patton MQ. Qualitative evaluation and research methods. Newbury Park, CA: Sage, 1990.

25. Buvik K, Rossow I. Factors associated with over-serving at drinking establishments. Addiction 2015;110:602-9. 
26. Toomey TL, Erickson DJ, Lenk KM, et al. A randomized trial to evaluate a management training program to prevent illegal alcohol sales. Addiction 2008;103:405-13; discussion 414-405.

27. Wallin E, Gripenberg J, Andréasson S. Overserving at licensed premises in Stockholm: effects of a community action program. J Stud Alcohol 2005;66:806-14.
28. Gripenberg J, Wallin E, Andréasson S. Effects of a communitybased drug use prevention program targeting licensed premises. Subst Use Misuse 2007;42:1883-98.

29. Gripenberg Abdon J, Wallin E, Andréasson S. Long-term effects of a community-based intervention: 5 year follow-up of "Clubs against Drugs". Addiction 2011;106:1997-2004. 